\title{
First-principles study of GaAs nanowires
}

\author{
S. Cahangirov ${ }^{1}$ and S. Ciraci ${ }^{1,2, *}$ \\ ${ }^{1}$ UNAM, Institute for Materials Science and Nanotechnology, Bilkent University, Ankara 06800, Turkey \\ ${ }^{2}$ Department of Physics, Bilkent University, Ankara 06800, Turkey \\ (Received 24 December 2008; revised manuscript received 17 March 2009; published 28 April 2009)
}

\begin{abstract}
In this paper we present a detailed analysis of the atomic and electronic structures of GaAs nanowires using first-principles pseudopotential calculations. We consider six different types of nanowires with different diameters all grown along [111] direction, and we reveal interesting trends between cohesive energy and nanowire type with varying diameters. Generally, the average cohesive energy of nanowires with wurtzite stacking is higher than those with zinc-blende stacking for small diameters. We found that most of the bare nanowires considered here are semiconducting and continue to be semiconducting upon the passivation of surface dangling bonds with hydrogen atoms. However, the surface states associated with the surface atoms having two dangling bonds in zinc-blende stacking occur in the band gap and can decrease the band gap to change the nanowire from semiconducting to metallic state. These nanowires become semiconducting upon hydrogen passivation. Even if the band gap of some nanowires decreases with increasing diameter and hence reveals the quantum confinement effect, generally the band-gap variation is rather complex, and depends on the type and geometry, diameter, type of relaxation, and also whether the dangling bonds of surface atoms are saturated with hydrogen.
\end{abstract}

DOI: 10.1103/PhysRevB.79.165118

PACS number(s): $61.46 . \mathrm{Km}, 62.23 . \mathrm{Hj}, 73.22 .-\mathrm{f}$

\section{INTRODUCTION}

In searching to discover semiconductor materials and metallic interconnect for new generation miniaturized electronic devices, nanostructures have been a focus of attention. Electronic devices, such as transistors based on carbon nanotubes, ${ }^{1}$ attracted interest in nanowires. Rodlike Si nanowires have been fabricated ${ }^{2}$ with a diameter of $1.3-7 \mathrm{~nm}^{3}$ It has been shown that such $\mathrm{Si}$ nanowires can display metallic, semiconducting, and half-metallic properties depending on their functionalization. ${ }^{4}$ Being an alternative to silicon based microelectronics GaAs is one of the most important materials used in semiconductor physics. Due to the high electron mobility, GaAs always carried a potential of being used in high-speed electronic devices. GaAs/AlGaAs heterostructures $^{5}$ have served as media for the twodimensional electron-gas studies.

Similar to bulk crystals researcher have envisioned GaAs nanowires to be a potential alternative for Si nanowires. Recent advances in fabrication technology made it possible to grow GaAs nanowires. They are grown by metal catalysts in vapor-liquid-solid (VLS) mechanism. ${ }^{6}$ Generally, GaAs nanowires are grown along [111] direction in zinc-blende $(z b)$ structure, whereas nanowires with wurtzite $(w z)$ structure with diameter as small as $10 \mathrm{~nm}$ are also observed. ${ }^{7}$ Several models were developed to predict the transition radius from wurtzite to zinc-blende structure. ${ }^{8,9}$ Actually, there is no sharp transition but instead there are many different stacking configurations with very similar energies and more sophisticated models are needed to predict the ground-state configuration. Together with the crystal structure, surface facet structure is also an important parameter affecting the structural and electronic properties. Nanowires reported so

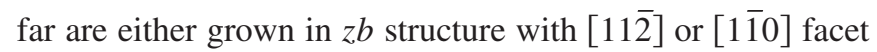

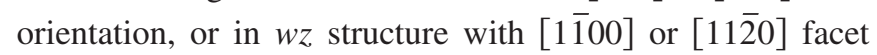
orientation. ${ }^{10-12}$ One of the unusual structures is so-called $A$ wire, which has been grown in defect free triangular shape on (111)A surface of GaAs. ${ }^{13}$ More complex structure of nanowires grown on $\operatorname{GaAs}(111) B$ surface has been also reported. ${ }^{14}$

There are many experimental and theoretical works on growth and structure of GaAs nanowires but not much work is done to predict the electronic properties of these wires. Theoretical studies so far have focused on the electronic properties of superlattices composed of GaAs nanowires. For example, the electronic structure of InAs/GaAs nanowire superlattices with radius $R=10 \mathrm{~nm}$ was examined using a semiempirical $s p^{3} d^{5} s^{*}$ tight-binding model. ${ }^{15}$ Another atomistic tight-binding calculation was carried out to reveal the electronic structure of freestanding $\mathrm{GaAs} / \mathrm{Al}_{0.3} \mathrm{Ga}_{0.7} \mathrm{As}$ nanowire superlattices oriented along the [100] crystallographic direction. ${ }^{16}$ Also a first-principles investigation has been performed on the hexagon-shaped [111]/[0001]-oriented III-V semiconductor nanowires, which was concentrated on the surface effects on the structure and stability of these nanowires. ${ }^{10}$

The purpose of this paper is to provide a detailed analysis of GaAs nanowires, which is necessary for further experimental and theoretical studies. To this end we present a systematic first-principles investigation on structural and electronic properties of GaAs nanowires grown along [111] direction. Six different types of GaAs nanowires are distinguished depending on the shape of their cross sections and the crystallographic orientation of their side surfaces. Their optimized atomic structure and cohesive energies are calculated revealing interesting trends between atomic structure and cohesive energy. Based on the calculations of electronic structure and isosurface charge density of specific states, we analyzed the character of states at the band edges and variation in band gap with diameter. The effects of hydrogen saturation of dangling bonds of surface atoms on the atomic and electronic structure are examined. We found that most of bare GaAs nanowires are semiconducting and remain semi- 
conducting even before the passivation of surface dangling bonds. Only one type is metallic due to the states localized at the surface.

\section{METHODS}

We have performed first-principles plane-wave calculations ${ }^{17,18}$ within density-functional theory (DFT) (Ref. 19) using ultrasoft pseudopotentials. ${ }^{18,20}$ The pseudopotentials having three electrons for $\mathrm{Ga}\left(4 s^{2} 4 p^{1}\right)$, five electrons for As $\left(4 s^{2} 4 p^{3}\right)$, and one electron for $\mathrm{H}\left(1 s^{1}\right)$ were used. A plane-wave basis set with kinetic energy up to $250 \mathrm{eV}$ was used. Cutoff energies used were at least 30\% higher than maximum values suggested. ${ }^{18}$ The exchange-correlation potential is approximated by generalized gradient approximation (GGA) using PW91 functional. ${ }^{21}$ For partial occupancies we use the Methfessel-Paxton smearing method. ${ }^{22}$ The adopted smearing width is $0.1 \mathrm{eV}$ for the atomic relaxation, and 0.01 for the accurate band-structure analysis and density of state calculations. All structures have been treated within a supercell geometry using the periodic boundary conditions. Vacuum spacing was arranged so that the minimum distance between two atoms in adjacent unit cells were larger than $10 \AA$, provided that atoms have negligible interaction at that far distances. We chose bare $w z 3-96$ as a test structure and increased the vacuum spacings to $16 \AA$. This resulted in an energy difference around $0.2 \mathrm{meV} /$ atom. In the selfconsistent potential and total-energy calculations the Brillouin zone (BZ) is sampled in the $\mathbf{k}$ space within MonkhorstPack scheme ${ }^{23}$ by $(1 \times 1 \times 9)$ mesh points for $w z$ and $(1$ $\times 1 \times 7)$ mesh points $z b$ nanowires. Increasing the $\mathbf{k}$-space sampling for bare $w z 3-96$ structure from $(1 \times 1 \times 9)$ to $(1$ $\times 1 \times 15)$ resulted in a total-energy difference around $1 \mathrm{meV}$. All atomic positions and lattice constant are optimized by using the conjugate gradient method where total energy and atomic forces are minimized. The criterion of convergence for energy is chosen as $10^{-5} \mathrm{eV}$ between two ionic steps, and the maximum force allowed on each atom is $0.05 \mathrm{eV} / \AA$. We have reduced the maximum force criterion down to $0.0025 \mathrm{eV} / \AA$ in our test structure, bare $w z 3-96$. This had no considerable effect since the change in energy and band gap was around $0.3 \mathrm{meV} / \mathrm{atom}$ and $0.006 \mathrm{eV}$, respectively. Clearly, the criterion for the maximum allowed force $0.05 \mathrm{eV} / \AA$ is appropriate for systems including large number of atoms.

\section{STRUCTURES AND COHESIVE ENERGIES}

GaAs nanowires studied here are cut from ideal bulk structure along [111] direction. Nanowires having wurtzite $(w z)$ and zinc-blende $(z b)$ stackings have four and six atomic layers in the unit cell, respectively. Except $A$ wire, they have hexagonal cross section. $A$ wires by themselves have $z b$ stacking and display a triangular cross section with three $(11 \overline{2})$ planar side surfaces. Atomic structures of cross section of all nanowires are shown before and after relaxation (structure optimization) in Fig. 1. Here we consider the largest members of all types of GaAs nanowires. Upon relaxation the surfaces of ideal wires undergo a reconstruction while
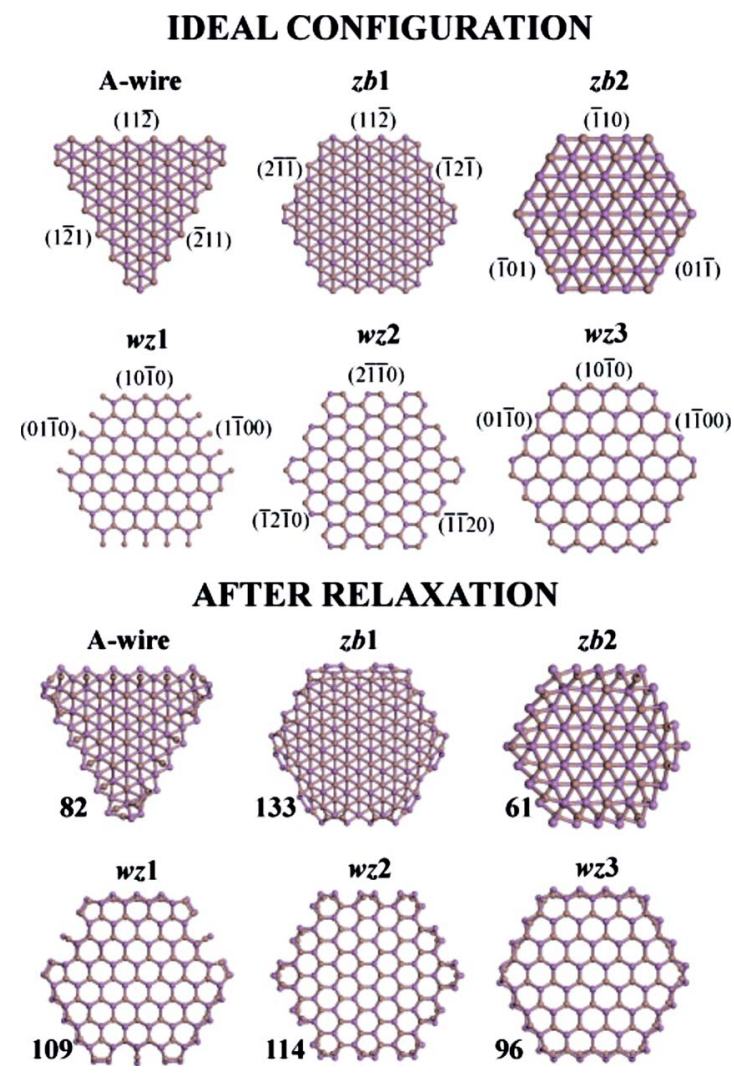

FIG. 1. (Color online) Ideal and relaxed atomic structures of bare GaAs nanowires considered in this paper. Numerals given in parenthesis indicate the crystallographic directions perpendicular to the surfaces. Numerals given to the bottom left of the structures stand for the number of atom pairs per unit cell $N . w z$ and $z b$ stand for structures having wurtzite and zinc-blende stackings.

inner parts preserve the bulk configuration. In spite of the fact that the indices of their planar side surface are the same, $w z 1$ and $w z 3$ are still different. $w z 3$ structure have six identical surfaces, whereas $w z 1$ structure have three surfaces same as $w z 3$ and three surfaces with hanging Ga-As atom pairs before relaxation.

At the surfaces of some ideal structures in Fig. 1 the atoms can have the coordination number smaller than four. Upon relaxation the coordination numbers may undergo a change. Two adjacent surface atoms having low coordination number can form new bonds, whereby these atoms increase their coordination number and the nanowire, in turn, lowers its energy (i.e., it becomes more energetic). It turns out that the coordination number of surface atoms is crucial for the value of the cohesive energy per atom pair. For example, $w z 1$ nanowire with $N=109$ has three surfaces each having an atom pair with coordination number of two while other three surfaces have surface atoms with coordination number of three. $w z 2$ nanowires having $N=25,60$, and 85 atom pairs in the unit cell have surface atoms with coordination number of two only at the corners of the hexagonal cross section. On the other hand, $w z 2$ nanowires having $N=42$ and 114 atom pairs in the unit cell, and all members of $w z 3$ nanowires have surface atoms with coordination number of three. In light of these arguments one expects $w z 3$ to have larger cohesive 


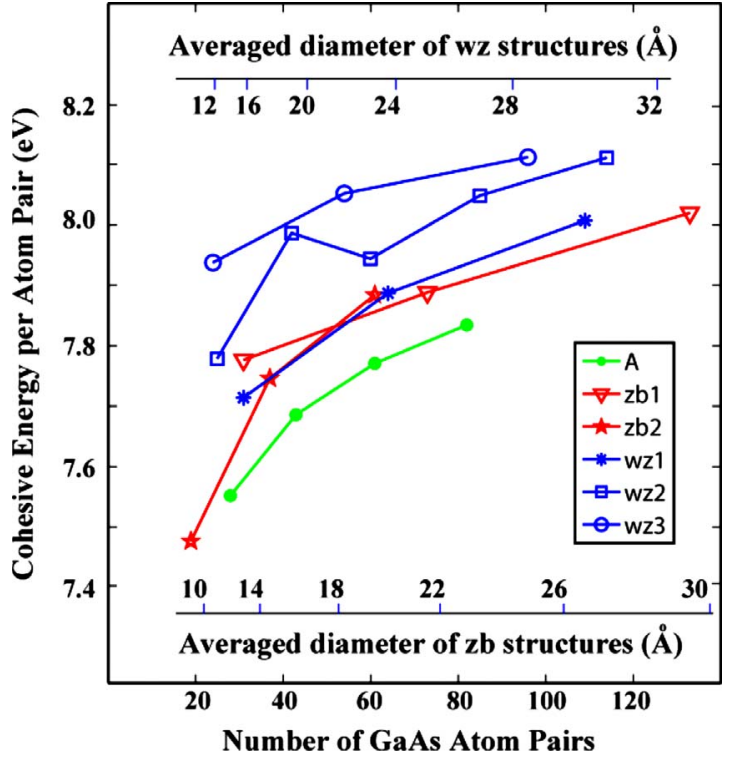

FIG. 2. (Color online) Cohesive energy per Ga-As atom pair versus number of Ga-As atom pairs in the unit cell of different type of relaxed nanowires. Horizontal axes presented inside the figure are derived by fitting the diameter versus number of atom data of $w z$ and $z b$ nanowires to quadratic polynomials. Since $w z$ and $z b$ structures have different number of atomic planes in the unit cell, the fitting was done separately.

energy per atom pair than the rest of $w z 2$ nanowires, which, in turn, should be larger than that of $w z 1$ nanowires. These arguments are actually confirmed in Fig. 2, where we present the trends of cohesive energies per atom pair for all nanowires considered here.

Ideal $A$ wires have surface atoms with coordination number of two on each surface, but apart from that, it has a triangular cross section, which makes the surface-to-volume ratio even higher compared to that of other types. Consequently, $A$ wires have the lowest cohesive energy per atom pair as seen in Fig. 2.

Interestingly, $w z$ structures have relatively larger cohesive energies than $z b$ structures, with $w z 3$ structure being the largest. The bulk $z b$ structure however is energetically more favorable than that of $w z$ structure by nearly $20 \mathrm{meV}$ per Ga-As pair, also confirmed by our calculations. As radii in- crease, the cohesive energy per Ga-As atom-pair values should converge to the bulk value. It is energetically easier to form a $w z$ surface than to form a $z b$ surface. In other words surface energy of $z b$ structure is larger. That is why, $w z$ structures become energetically more favorable as the surface-tovolume ration increases, namely, as the radius of the nanowire decreases. So there should be some point where cohesive energy of $w z$ and $z b$ structures cross each other. The radius at this point can be interpreted as the critical radius for transition from $w z$ to $z b$ structure or vice versa. Here it should be noted that energetically favorable does not mean that these structures will start to grow in experiments. One should also include effects of formation path, such as nucleation growth. Actually, when we are around the critical radius we can see some hybrid stacking patterns such as $A B A C$, which is defined as $4 H$ structure. ${ }^{9}$

Table I gives the calculated values for the structure and cohesive energies of nanowires after the relaxation. Cohesive energies per Ga-As atom pair increase with increasing diameter, approaching the bulk values, but we do not see the critical radius because it is expected to be an order-ofmagnitude larger than that of our nanowires. ${ }^{9}$ Surprisingly, the lattice constant decreases as the diameter of nanowires in $z b$ structure increases while for $w z$ structures the reverse situation occurs. The ratio of number of surface atoms to the total number of atom pairs give a measure of surface-tovolume ratio, which is decreasing with increasing diameter. Note that the number of surface atoms having coordination number of two is $\left(N_{D}-N_{S}\right)$.

\section{ELECTRONIC STRUCTURE}

Most of the relaxed GaAs nanowires presented in Fig. 1 are semiconducting even without hydrogen saturation. As we will see, in some cases these bare GaAs nanowires do not even have the surface states at the band edges. This situation is in contrast with $\mathrm{Si}$ nanowires. ${ }^{2-4,24,25} \mathrm{Si}$ nanowires as cut from the bulk crystal and subsequently relaxed are found to be metallic. Their metallicity occurs due to the partial filling of the dangling bonds surface states. Upon passivation of the dangling bonds with hydrogen atoms the surface states are discarded from the band gap and eventually Si nanowire becomes semiconductor. In what follows, we will examine the

TABLE I. Calculated cohesive energy per Ga-As atom pair $E_{c}$, band gap $E_{g}$, lattice constant along the wire axis $c$, and diameter $D$ values of relaxed nanowires are given. $D$ is defined as the largest distance between two atoms in the same cross-sectional plane. Here $N$ stands for the number of Ga-As atom pairs in the unit cell. $N_{S}$ and $N_{D}$ stand for the number of surface atoms and the total number of dangling bonds, respectively. Surface atoms are defined as atoms making less than four bonds while the protruding bonds are defined as dangling bonds.

\begin{tabular}{|c|c|c|c|c|c|c|c|c|c|c|c|c|c|c|c|c|c|c|c|c|c|}
\hline \multirow{2}{*}{$\frac{\text { Type }}{N}$} & \multicolumn{3}{|c|}{$w z 1$} & \multicolumn{5}{|c|}{$w z 2$} & \multicolumn{3}{|c|}{$w z 3$} & \multicolumn{4}{|c|}{$A$} & \multicolumn{3}{|c|}{$z b 1$} & \multicolumn{3}{|c|}{$z b 2$} \\
\hline & 31 & 64 & 109 & 25 & 42 & 60 & 85 & 144 & 24 & 54 & 96 & 28 & 43 & 61 & 82 & 31 & 73 & 133 & 19 & 37 & 61 \\
\hline$E_{c}(\mathrm{eV})$ & 7.71 & 7.89 & 8.01 & 7.78 & 7.99 & 7.94 & 8.05 & 8.11 & 7.94 & 8.05 & 8.11 & 7.55 & 7.69 & 7.77 & 7.83 & 7.78 & 7.89 & 8.02 & 7.48 & 7.75 & 7.88 \\
\hline$E_{g}(\mathrm{eV})$ & 1.06 & 1.02 & 0.92 & 0.81 & 0.96 & 0.95 & 0.92 & 0.88 & 1.45 & 1.10 & 0.90 & 0.81 & 0.77 & 0.85 & 0.70 & 1.07 & 0.14 & 0.58 & $\mathrm{M}$ & $\mathrm{M}$ & $\mathrm{M}$ \\
\hline$c(\AA)$ & 6.64 & 6.63 & 6.63 & 6.58 & 6.59 & 6.61 & 6.61 & 6.63 & 6.60 & 6.61 & 6.63 & 9.94 & 9.89 & 9.88 & 9.87 & 9.97 & 9.95 & 9.85 & 9.97 & 9.87 & 9.84 \\
\hline$D(\AA)$ & 15.9 & 23.6 & 31.6 & 14.5 & 18.1 & 23.1 & 28.4 & 32.0 & 12.5 & 20.5 & 28.6 & 12.6 & 16.3 & 19.8 & 24.5 & 12.7 & 20.9 & 29.0 & 9.2 & 14.5 & 18.3 \\
\hline$N_{S}$ & 24 & 36 & 48 & 24 & 36 & 36 & 48 & 60 & 24 & 36 & 48 & 29 & 36 & 45 & 54 & 30 & 48 & 66 & 24 & 36 & 48 \\
\hline$N_{D}$ & 30 & 36 & 54 & 27 & 36 & 43 & 51 & 60 & 24 & 36 & 48 & 38 & 42 & 54 & 66 & 30 & 54 & 66 & 30 & 42 & 54 \\
\hline
\end{tabular}




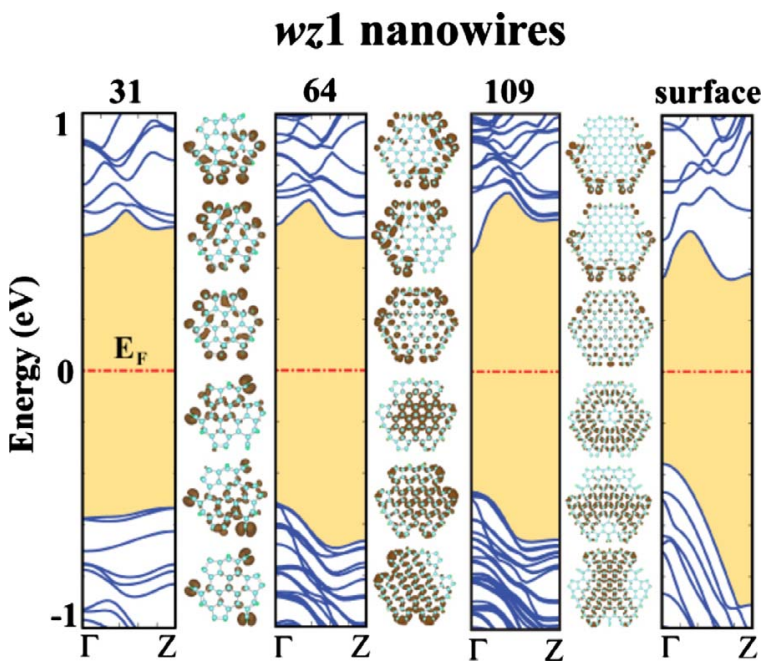

FIG. 3. (Color online) Band structure and charge-density isosurface plots of GaAs nanowires having wz1 structure. Energy band gap between the valence and conduction bands is shaded. Numerals given on top of the bands stand for the number of GaAs atom pairs $N$ in the unit cell. Charge density isosurfaces of specific states at $\Gamma$ point are shown on the right-hand side of the bands they belong to. Isosurface charge densities correspond to three valence-band and three conduction-band edge states, ordered in the same manner as bands themselves are. Here we also give the band structure of infinite slab of bulk wurtzite structure consisting 11 atomic layers with the same planar $(10 \overline{1} 0)$ surfaces as $w z 1$ nanowires does. Zero of energy is set at the Fermi level $E_{F}$.

electronic structure of bare GaAs nanowires, and reveal the effects of geometry and the passivation of dangling bonds with hydrogen. ${ }^{26}$

Figure 3 presents results of the band structure and chargedensity analysis performed for $w z 1$ structure. Ideal structure of these wires have Ga-As atom pairs hanging on three surfaces while other three surfaces have the same profile as $w z 3$ type. Upon relaxation these hanging pairs tend to bend toward each other, and lower energy by making unusual $\mathrm{Ga}-\mathrm{Ga}$ and As-As bonds. For example, wz1 structure having 64 atom pairs per unit cell $(w z 1-64)$ have four hanging pairs before relaxation. After relaxation first two and last two of them bend to each other, and form a stable structure. $w z 1-31$ and wz1-109 nanowires have one hanging pair after relaxation. As a result, all surface atoms of $w z 1-64$ structure have coordination number of three while $w z 1-31$ and $w z 1-109$ have six surface atoms with coordination number of two (see Table I). Isosurface charge densities show that three states at the top of the valence-band edge of $w z 1-64$ and $w z 1-109$ structures have bulklike character while conduction-band edge states concentrate at the surface. It is hard to define the nature of states in $w z 1-31$ structure because it has low diameter. To calculate the band structure of the relevant surface we cut a slab from the bulk wurtzite structure parallel to (01) 10$)$ surfaces so that the resulting structure have 11 atomic layers. This slab has two-dimensional periodicity in the surface and a vacuum region between adjacent surfaces so that they do not interact. The band structure of this slab plotted along $\mathbf{k}$, parallel to [111] direction, provide us with informa-

\section{wz2 nanowire}
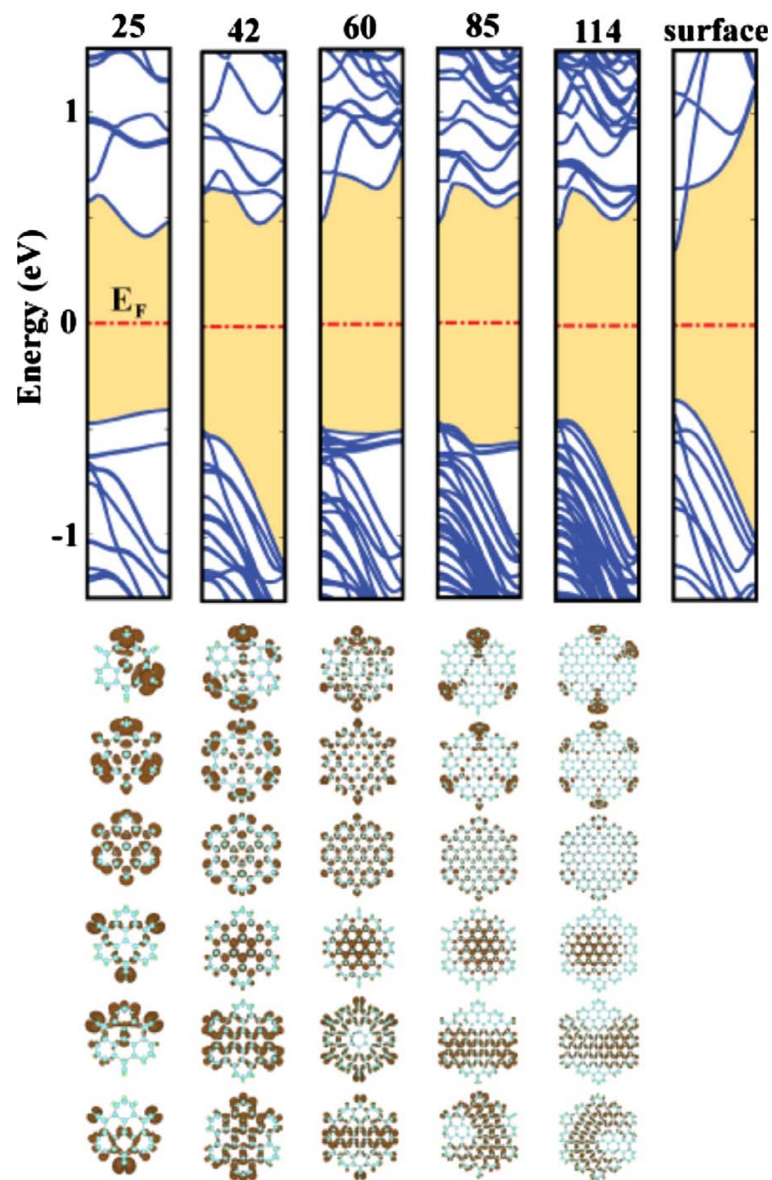

FIG. 4. (Color online) Same as in Fig. 3 but for $w z 2$ structure. Isosurface charge densities correspond to three valence-band and three conduction-band edge states, ordered in the same manner as bands themselves are.

tion about the band structure of nanowires if they were grown thick enough to have a reasonable bulk region in order to reduce the corner effects. One expects the band gap of the infinite slab structure to be lower than that of the nanowires. Comparison of the bands of $w z 1-109$ with those of

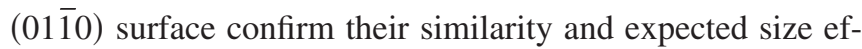
fect.

Figure 4 shows the band structure and charge-density analysis for $w z 2$ structures. Here all surface atoms of $w z 2-42$ and $w z 2-114$ structures have coordination number of three while the rest of considered $w z 2$ structures have surface atoms with coordination number of two at the corners. Note that the valence-band edge of $w z 2-42$ and $w z 2-114$ structures mimic that of infinite slab structure while other structures fail to do that. We see that the band gap of $w z 2-42$ is larger than that of $w z 2-114$ structure in agreement with the quantum confinement effect. On the other hand, however, $w z 2-25$ structure have the lowest band gap. This is caused by large surface-to-volume ratio, which make surface effects pronounced. Isosurface charge densities show that the valence-band edge has bulklike character while conduction-band edge states concentrate on the surface. 


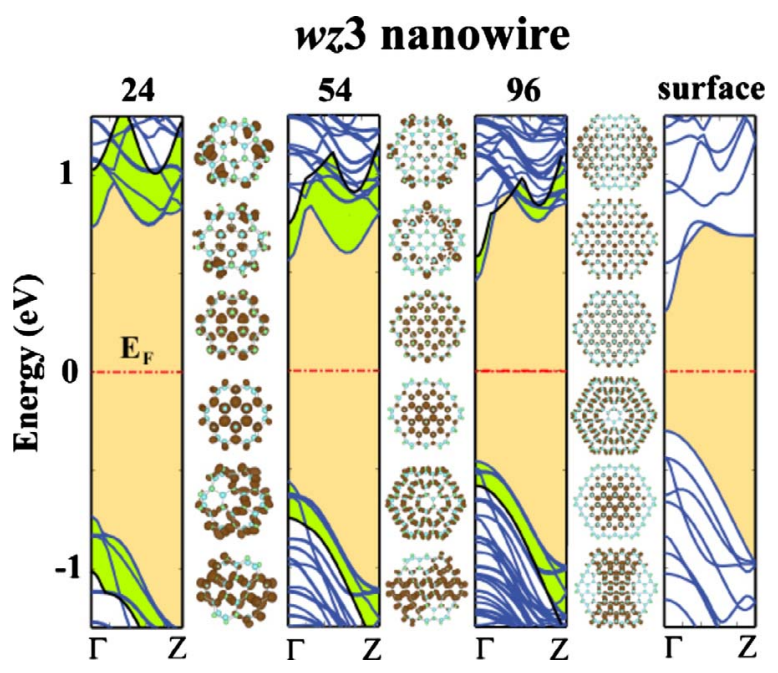

FIG. 5. (Color online) Band structure and charge-density isosurface plots of GaAs nanowires having wz3 structure. Energy band gap between the valence and conduction bands of bare nanowire is (yellow) light shaded. Numerals given on top of the bands stand for the number of GaAs atom pairs $N$ in the unit cell. Charge density isosurfaces of specific states at $\Gamma$ point are shown on the right side of the bands they belong to. Isosurface charge densities correspond to three valence-band and three conduction-band edge states, ordered in the same manner as bands themselves are. Here we also give the band structure of infinite slab of bulk wurtzite structure consisting 11 atomic layers with the same planar (10 $\overline{1} 0)$ surfaces as $w z 3$ nanowires does. Zero of energy is set at the Fermi level $E_{F}$. The widening of the band gap upon the termination of dangling bonds by hydrogen is shown by (green) dark-shaded regions delineated by black curves at the valence- and conduction-band edges.

Band structure and charge-density analysis of $w z 3$ structure is illustrated in Fig. 5. This structure has the largest cohesive energy per atom value compared to other types of nanowires in Fig. 1. In this nanowire all surface atoms make three bonds and surface states derived therefrom do not occur in the band gap. Consequently, the band structure of the related infinite slab is very similar to that of $w z 3-96$ nanowire. Charge density of the states at both band edges are spread throughout the nanowire cross section showing the bulk character. Since band gap is not diminished by surface states bands, one can clearly see the quantum confinement effect in these nanowires.

Passivation of dangling bonds of semiconducting nanowires by hydrogen atoms, generally, results in significant changes in the electronic structure. These changes depend on whether the passivation is done before or after the relaxation of bare nanowires. We find the latter case more suitable for the simulation of the experimental procedure. ${ }^{4}$ Figure 5 includes information about the effect of hydrogen passivation of all surface dangling bonds on the band structure of $w z 3$ nanowires. In contrast to Si nanowires (where surface states of bare structure were carried out from forbidden region to the band continua upon passivation with hydrogen), ${ }^{4,27,28}$ the band-edge states of $w z 3 \mathrm{GaAs}$ nanowires remain in their place after the hydrogen saturation. This conclusion is corroborated by the analysis of isosurface charge density of states at the center of BZ located at both edges of band gap. wz3-96 nanowire
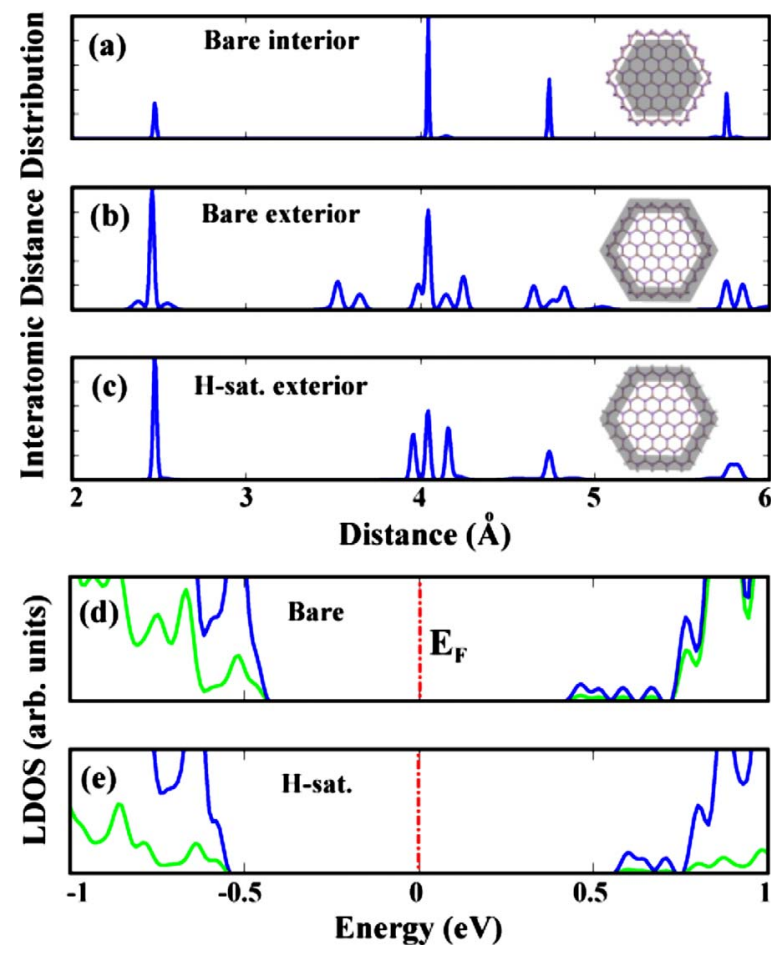

FIG. 6. (Color online) Interatomic distance distribution of the core and shell parts of bare and hydrogen saturated $w z 3-96$ structures. The ball and stick model illustrates the structure of $w z 3-96$ nanowire while the shaded regions defines the core and shell parts of the nanowires. (a) Interatomic distance distribution of interior atoms of bare nanowire. (b) Interatomic distance distribution of exterior atoms of bare nanowires. (c) Interatomic distance distribution of hydrogen passivated exterior atoms. (d) Local density of states on surface atoms (green/light) and on the remaining atoms (blue/dark) of bare nanowire. (e) Same as (d) after passivation of surface atoms with hydrogen.

We found that the character and charge distribution of these states do not undergo a change after passivation of surface dangling bonds with hydrogen atoms. Also the similarity in the profile of band edges before and after hydrogen saturation is found to be striking. Hydrogen atoms mostly affect the surface states, which, in $w z 3$ structure, are found in the valence-band continua. That is why the effect of hydrogen saturation is not reflected on the band edges in the way it was in silicon nanowires having surface states at the band edges. Here the increase in the band gap occurs not because the edge states are cleared out but because the atomic structure of the nanowires become more bulklike. This effect is illustrated in Fig. 6, where we analyzed the interatomic distance distribution of $w z 3-96$ structure before and after the hydrogen saturation. Plots given here are done by making a histogram of interatomic distances and then smearing it out. The first plot indicates the interatomic distance distribution of the core region of bare nanowire. We get the same result also in the core region of hydrogen saturated structure, and the peaks match the first, second, third, and fourth nearestneighbor distances of bulk GaAs in $w z$ structure. The crucial difference between bare and hydrogen saturated structures is 


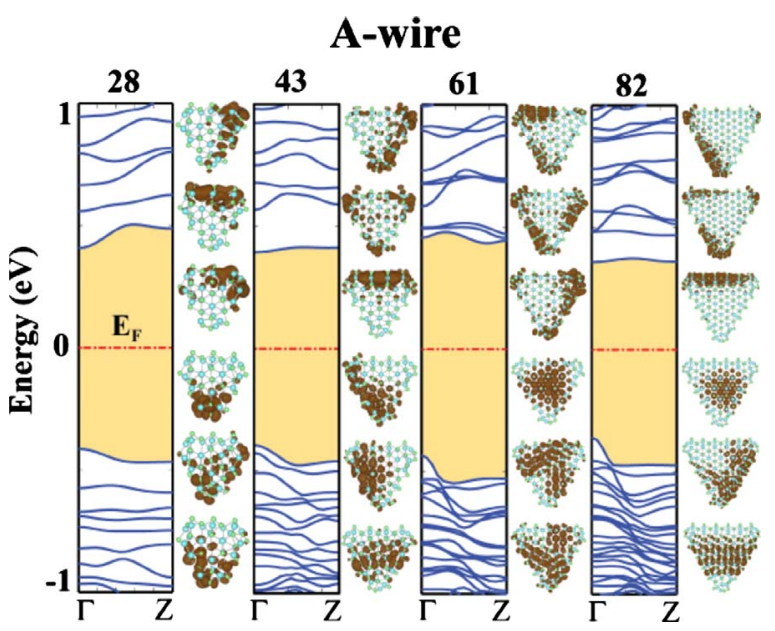

FIG. 7. (Color online) Band structure and charge-density isosurface plots for $A$ wires.

reflected in the shell part of the nanowires. One can clearly see that hydrogen saturated structure have more bulklike character.

Furthermore we performed atom projected density-ofstates analysis by calculating the localized density of states on the surface atoms, as well as on the core atoms before and after passivation with hydrogen atoms. As seen in Figs. 6(d) and 6(e), surface as well as core atoms have comparable contributions to the state distribution at both edges of band gap. These results further corroborate the fact that GaAs nanowires such as wz3-96 having surface atoms with coordination number of three do not have dangling-bond surface states in the band gap. For nanowires with large $D$ the passivation with hydrogen have negligible effects on the band gap. Note that the increase in the band gap with decreasing diameter holds also for hydrogen saturated structures.

In Fig. 7 we present the band structures and isosurface charge densities of selected states at $\Gamma$ point of $A$ wires. Note that we cannot have an infinite slab corresponding to the large $A$ wires because these nanowires have triangular cross section. All relaxed $A$ wires have surface atoms with coordination number of two. The band gap have no obvious trend with varying diameter and is determined by surface states especially falling in the conduction-band edge. For small $D$ the edges of band gap are determined by surface states with low dispersion. As the diameter increases the valence-band edge starts to show bulk character while states at the conduction-band edge remain to display surface character. Even for $A-82$ nanowire the flatband states at the edge of the conduction band are located on one of the planar side surface.

The effect of surface states on the band gap is dramatic for the bare GaAs nanowires having $z b$ stacking. The values of band gap as large as $1.5 \mathrm{eV}$ occurring in $w z$-type nanowires reduce to the values as small as $\sim 0.2 \mathrm{eV}$ in $z b 1$-type nanowires. The calculated band gap can even be closed in $z b 2$-type nanowires. It appears that many of the danglingbond surface states, in particular those associated with the surface atoms having coordination number of two, are placed in the band gap. Therefore one expects dramatic changes in

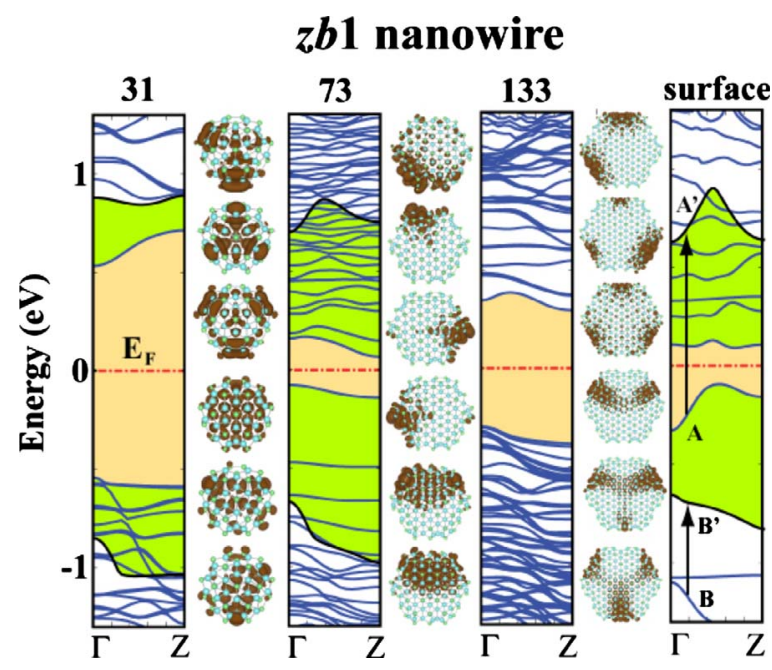

FIG. 8. (Color online) Band structure and charge-density isosurface plots of GaAs nanowires having zb1 structure. Energy band gap between the valence and conduction bands of bare nanowire is (yellow) light shaded. Numerals given on top of the bands stand for the number of GaAs atom pairs $N$ in the unit cell. Charge density isosurfaces of specific states at $\Gamma$ point are shown on the right-hand side of the bands they belong to. Isosurface charge densities correspond to three valence-band and three conduction-band edge states, ordered in the same manner as bands themselves are. Here we also give the band structure of infinite slab of bulk wurtzite structure with the same planar $(11 \overline{2})$ surfaces as $z b 1$ nanowires does. Zero of energy is set at the Fermi level $E_{F}$. The widening of the band gap upon the termination of dangling bonds by hydrogen is shown by (green) dark-shaded regions for $N=31,73$, and surface.

the electronic properties after the passivation of surface dangling bonds with hydrogen atoms. The electronic structures of bare $z b 1$-type nanowires are illustrated in Fig. 8. Here all surface atoms of $z b 1-31$ and $z b 1-113$ nanowires have coordination number of three after relaxation but certain surface atoms of $z b 1-73$ structure cannot find a pair to form a bond and stay with coordination number of two. As a result the band gap of $z b 1-73$ does not have a value between that of $z b 1-31$ and $z b 1-133$, as one would expect. To get more information about the nature of the states at both edges of the band gap, we have saturated all dangling bonds of $z b 1-31$ and $z b 1-73$ structures by hydrogen. After the hydrogen saturation the band-edge profile does not stay the same as it was in the case of $w z 3$ structures. This means that the band gap of bare nanowire has increased upon hydrogen passivation of dangling bonds since the band gap is cleared from the surface states. Apparently, the band-edge states of the bare $z b 1$ nanowire originate from the surface while in $w z 3$ structure they have bulklike behavior. The different behavior of triply coordinated surface atoms in $z b 1$ and $w z 3$ structure is attributed to their structural orientation. Surface atoms of $z b 1$ structure make atomic rings perpendicular to the wire axis. Dangling bonds of these atomic rings form several minibands (with low dispersion along $\Gamma Z$ ) in the band gap, with charge density localized at the surface. Surface atoms of $w z 3$ structure, however, form atomic chains along the wire axis. The dangling bonds of these atomic chains form surface states with high dispersion, which fall in the band continua. 


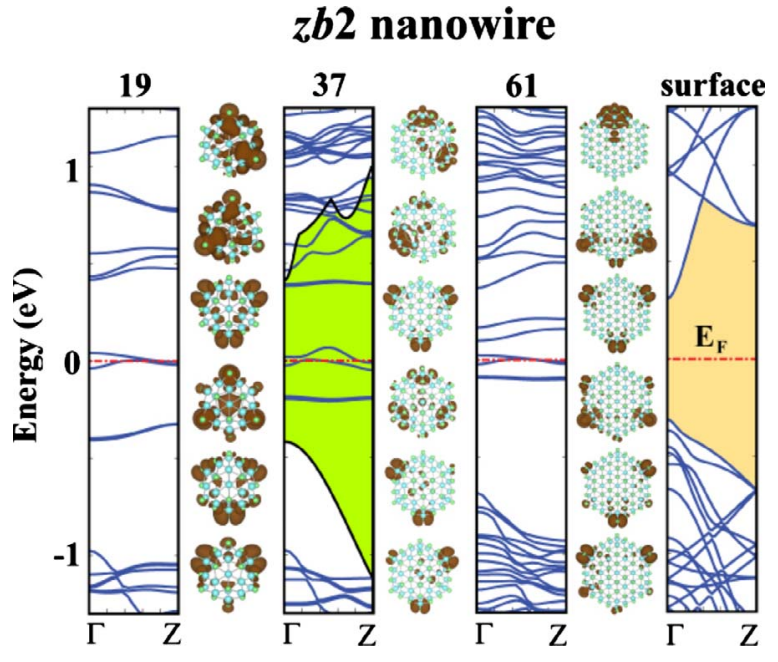

FIG. 9. (Color online) Same as in Fig. 8 but for $z b 2$ structure. The shaded region in the band structure of $z b 2-37$ corresponds to the band gap opened after the passivation of dangling bonds with hydrogen. $z b 2-19$ and $z b 2-61$ nanowires are not passivated with hydrogen

The effect of hydrogen saturation is investigated in the case of a slab in Fig. 8. Here the first and third valence-band edge states of bare slab structure are labeled as A and B, respectively. These dispersive states have bulklike character. Upon the passivation band $\mathrm{A}$ rises to $\mathrm{A}^{\prime}$ forming the conductionband edge, and band $\mathrm{B}$ rises to $\mathrm{B}^{\prime}$ forming the valence-band edge of the passivated structure. Surface states having low dispersion are lowered to valence-band continua.

The effects of the dangling-bond surface states are even more dramatic in zb2-type bare GaAs nanowires in Fig. 9, where the band gaps are closed and the nanowires become metallic. Therefore, $z b 2$ type is the only type we have considered to have metallic character. It is interesting that the corresponding infinite slab structure have a finite band gap. $z b 2$ structures would have a band gap which is larger than that of the slab structure if they would had no partly filled corner states crossing the Fermi level. Isosurface chargedensity plots support the idea of band-edge states being originated from the dangling bonds at the corners of hexagonal cross section. We further tested these arguments by passivating the dangling bonds of $z b 2-37$ with hydrogen. Upon hydrogen passivation all flat surface-state bands in the range of energy from $-0.5 \mathrm{eV}$ to $0.5 \mathrm{eV}$ disappeared and a band gap of $0.9 \mathrm{eV}$ opened. The resulting band profiles near the edges of conduction and valence bands are similar to those of the (110) slab surface given at the right-hand side of Fig. 9.

\section{DISCUSSIONS AND CONCLUSIONS}

We have performed first-principles DFT calculations to reveal the atomic and electronic structures of six different types of bare and hydrogen saturated GaAs nanowires. Nanowires considered have a diameter less than $3 \mathrm{~nm}$, and at this sizes $w z 3$ type have the highest cohesive energy per atom pair while $A$ wires have the lowest. In general, $w z$ nanowires have higher cohesive energy than $z b$ nanowires but the difference in cohesive energies decrease with increasing diameter.

We found that all bare GaAs nanowires are semiconducting except $z b 2$ type. In the latter structure, dangling-bond states of atoms having coordination number of two cross the Fermi level and hence the structure becomes metallic. For bare GaAs nanowires in $w z$ structure with surface atoms all having coordination number of three, the dangling-bond states associated with these surface atoms do not appear in the band gap but rather in the band continua. Therefore, quantum (size) confinement effect is apparent in their bandgap variation with radius. For these $w z$-type nanowires the band gap may increase upon the passivation of dangling bonds with hydrogen since bonding of surface atoms become more bulklike.

Surface states of GaAs nanowires in $z b$ structure fall in the band gap, even if all surface atoms have coordination number of three. In $w z$ structure surface atoms form atomic chains along the wire axis while in $z b$ structure they form noninteracting atomic rings perpendicular to the wire axis. Hydrogen saturation dramatically increases the band gap of $z b$ structures by clearing the surface states from the forbidden region. Generally, increasing diameter results in more bulklike valence-band edge; however conduction-band edge behaves more surfacelike.

For reasons explained in detail, the band-gap variation in GaAs nanowires is rather complex, and depends on their type and geometry, diameter, relaxation, and also whether the dangling bonds of surface atoms are passivated with hydrogen. We believe that present results are valuable for further research on GaAs and other III-V compound nanowires dealing with their doping, forming heterostructure and multiple quantum well structure, and their fuctionalization to get new electronic and magnetic properties. ${ }^{29,30}$

\section{ACKNOWLEDGMENTS}

Part of the computations have been carried out by using UYBHM at Istanbul Technical University through a grant (Grant No. 2-024-2007).

\footnotetext{
*ciraci@fen.bilkent.edu.tr

${ }^{1}$ See for a recent review of the subject: S. Ciraci, S. Dag, T. Yildirim, T. Senger, and O. Gulseren, J. Phys.: Condens. Matter 16, R901 (2004).
}

${ }^{2}$ J. Hu, T. W. Odom, and C. M. Lieber, Acc. Chem. Res. 32, 435 (1999).

${ }^{3}$ D. D. D. Ma, C. S. Lee, F. C. K. Au, S. Y. Tong, and S. Lee, Science 299, 1874 (2003). 
${ }^{4}$ E. Durgun, D. Cakir, N. Akman, and S. Ciraci, Phys. Rev. Lett. 99, 256806 (2007); E. Durgun, N. Akman, and S. Ciraci, Phys. Rev. B 78, 195116 (2008).

${ }^{5}$ L. Esaki and L. L. Chang, Phys. Rev. Lett. 33, 495 (1974).

${ }^{6}$ J. C. Harmand, G. Patriarche, N. Péré-Laperne, M.-N. MératCombes, L. Travers, and F. Glas, Appl. Phys. Lett. 87, 203101 (2005).

${ }^{7}$ S. O. Mariager, C. B. Sørensen, M. Aagesen, J. Nygård, and R. Feidenhans'1, Appl. Phys. Lett. 91, 083106 (2007).

${ }^{8}$ F. Glas, J. C. Harmand, and G. Patriarche, Phys. Rev. Lett. 99, 146101 (2007).

${ }^{9}$ V. G. Dubrovskii and N. V. Sibirev, Phys. Rev. B 77, 035414 (2008).

${ }^{10}$ R. Leitsmann and F. Bechstedt, J. Appl. Phys. 102, 063528 (2007).

${ }^{11}$ K. Hiruma, M. Yazawa, K. Haraguchi, and K. Ogawa, J. Appl. Phys. 74, 3162 (1993).

${ }^{12}$ B. J. Ohlsson, M. T. Björk, M. H. Magnusson, K. Deppert, and L. Samuelson, Appl. Phys. Lett. 79, 3335 (2001).

${ }^{13}$ B. A. Wacaser, K. Deppert, L. S. Karlsson, L. Samuelson, and W. Seifert, J. Cryst. Growth 287, 504 (2006).

${ }^{14}$ L. S. Karlsson, K. A. Dick, J. B. Wagner, J. O. Malm, K. Deppert, L. Samuelson, and L. R. Wallenberg, Nanotechnology 18, 485717 (2007).

${ }^{15}$ Y. M. Niquet, Phys. Rev. B 74, 155304 (2006).

${ }^{16}$ M. P. Persson and H. Q. Xu, Phys. Rev. B 73, 035328 (2006).

${ }^{17}$ M. C. Payne, M. P. Teter, D. C. Allen, T. A. Arias, and J. D. Joannopoulos, Rev. Mod. Phys. 64, 1045 (1992).
${ }^{18}$ Numerical computations have been carried out by using VASP software: G. Kresse and J. Hafner, Phys. Rev. B 47, 558 (1993); G. Kresse and J. Furthmuller, ibid. 54, 11169 (1996).

${ }^{19}$ W. Kohn and L. J. Sham, Phys. Rev. 140, A1133 (1965); P. Hohenberg and W. Kohn, Phys. Rev. 136, B864 (1964).

${ }^{20}$ D. Vanderbilt, Phys. Rev. B 41, 7892 (1990).

${ }^{21}$ J. P. Perdew, J. A. Chevary, S. H. Vosko, K. A. Jackson, M. R. Pederson, D. J. Singh, and C. Fiolhais, Phys. Rev. B 46, 6671 (1992).

${ }^{22}$ M. Methfessel and A. T. Paxton, Phys. Rev. B 40, 3616 (1989).

${ }^{23}$ H. J. Monkhorst and J. D. Pack, Phys. Rev. B 13, 5188 (1976).

${ }^{24}$ P. Sen, O. Gulseren, T. Yildirim, I. P. Batra, and S. Ciraci, Phys. Rev. B 65, 235433 (2002).

${ }^{25}$ R. Rurali and N. Lorente, Phys. Rev. Lett. 94, 026805 (2005).

${ }^{26}$ Caution has to be taken in the band-gap values calculated in this study since DFT underestimates their values. For example, present calculations predict the band gap of bulk GaAs to be only $0.27 \mathrm{eV}$ as compared to the experimental value of $1.4 \mathrm{eV}$.

${ }^{27}$ P. W. Leu, B. Shan, and K. Cho, Phys. Rev. B 73, 195320 (2006).

${ }^{28}$ M. Nolan, S. O'Callaghan, G. Fagas, J. C. Greer, and T. Frauenheim, Nano Lett. 7, 34 (2007).

${ }^{29}$ M. S. Gudiksen, L. J. Lauhon, J. Wang, D. C. Smith, and C. M. Lieber, Nature (London) 415, 617 (2002).

${ }^{30}$ M. T. Bjork, B. J. Ohlsson, T. Sass, A. I. Persson, C. Thelander, M. H. Magnusson, K. Deppert, L. R. Wallenberg, and L. Samuelson, Nano Lett. 2, 87 (2002). 